\title{
Die Leiden der jungen Clara: \\ Das Klaviertrio Opus 17 als Ausdruck einer Neu-Romantikerin
}

SIEGWART REICHWALD, SPARTANBURG/SOUTH CAROLINA

\section{Einleitung}

"The G minor Finale [...] shows Clara's craftsmanship at its peak. Not only can the first theme be regarded as a subtle metamorphosis of the opening phrase of the Andante, but in the development section its own rhythm is changed into a stern, quasi fugal subject which, juxtaposed with the second subject, is explored with contrapuntal cunning of which Mendelssohn would not have been ashamed even if a little too academic for Schumann."

Dieses Zitat von Joan Chissell ist emblematisch für den Stand der Clara Schumann Forschung. Bücher, Dissertationen und Aufsätze haben zwar schon einiges über Schumann als Komponistin erschlossen, aber Studien, die einzelne Werke tiefgründiger interpretieren, sind nur sehr begrenzt vorhanden.

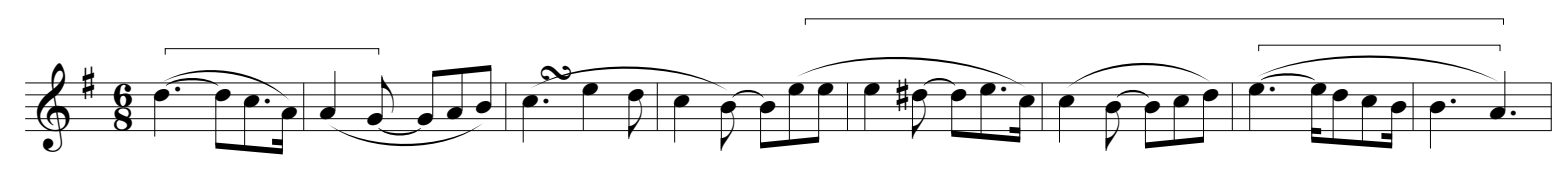

Notenbeispiel 1: Clara Schumann, Op. 17, dritter Satz, Hauptthema

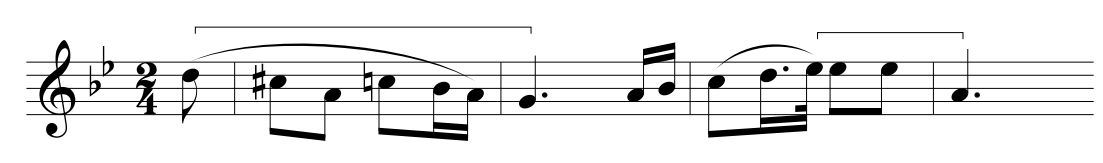

Notenbeispiel 2: Clara Schumann, Op. 17, vierter Satz, Hauptthema

Chissells Beschreibung der Metamorphose ist leicht nachzuvollziehen, und wir können zwei übergreifende diastematische Merkmale erkennen. Die fallende Quinte von Dominante zum Grundton und die verminderte bzw. reine Quinte von der sechsten zur zweiten Stufe. Die Metamorphose führt uns von Dur zu Moll und Quinte zum Tritonus, also nicht von Nacht zum Licht oder Leid zur Erlösung. Allerdings offeriert Chissell dafür keine Erklärung.

1 Joan Chissell, Clara Schumann: A Dedicated Spirit: A Study of her Life and Work, London 1983, S. 97. 
Zeitgenössische Rezensionen sind sich wohl dieses ungewöhnlichen Vorgehens bewusst, denn sie weisen insbesondere auf den melancholischen Charakter dieses Trios hin. Hermann Krüger hört in der Komposition "etwas Melancholisches und nur dann und wann einige lichtere Stellen, die um so mehr wirkten, da das Ganze in einen Schleier gehüllt erscheint", ${ }^{2}$ und für Flodoard Geyer ist „[d]as ganze Werk [...] von einer wehmütigen, stillen Trauer umflossen“3. Clara Schumann wurde in der Presse als Komponistin ernst genommen und zu den fortschrittlichen Komponisten gezählt. Anhand des Autographen, damaliger Rezensionen, und harmonischer sowie motivischer Analyse können wir dieses Werk biographisch und stilistisch kontextualisieren und damit hermeneutische Einblicke gewinnen. Dabei werden wir feststellen, wie fortschrittlich dieses Werk wirklich ist. Clara Schumann hat als Romantikerin eine Reihe von progressiven Mitteln genutzt, um musikalisch ihre schwierigen Erfahrungen in Dresden aufzuarbeiten.

\section{Deprimierendes Dresden}

Dresden sollte ein Neubeginn sein: Versöhnung mit ihrem Vater, ein neues Publikum, Roberts Genesung und ein dynamisches musikalisches Umfeld. Jedoch war Friedrich Wieck weiterhin feindlich gesinnt, Roberts Musik war überall höher geschätzt als in Dresden, und seine Depression vertiefte sich, was zu gesellschaftlicher Isolierung führte. Außerdem waren Konzertreisen nicht mehr im gewohnten Umfang möglich: Zur Zeit des Umzuges im Dezember 1844 war Clara im sechsten Monat schwanger, und in den nächsten zweieinhalb Jahren wurden zwei weitere Kinder geboren. Musikalisch war die Isolierung umso härter, denn Dresden hatte kein Gewandhaus, kein Konservatorium und keine gleichgesinnten Persönlichkeiten auf internationalem Niveau wie Felix Mendelssohn. Richard Wagner und die neue Semperoper vertraten andere musikalische Ideale. Ihr kleiner musikalischer Kreis bestand lediglich aus Ferdinand Hiller, Wilhelmine Schröder-Devrient und den Brüdern Franz und Friedrich Schubert. Auf der anderen Seite aber wurden Freundschaften gesucht mit Malern und Schriftstellen, was natürlich die breitere Basis ihres Musikverständnisses bereicherte.

Clara und Robert stellten sich der neuen Sachlage mit Entschlossenheit. Im Frühjahr 1845 studierten sie Kontrapunkt, und Clara veranstaltete Kammermusik-Soireen. Zusammen mit den Schubert Brüdern wurden u. a. Klaviertrios aufgeführt. Clara Schumann sah sich nicht in erster Linie als Komponistin. Der Konzertsaal war ihre Domäne. Aber ihr musikalisches Vermächtnis ist nicht Virtuosität, sondern Interpretation. Schon als 18-Jährige wurde ihr fortschrittliches Vorgehen von der Presse erkannt, wenn diese sie sogar als Mitglied der neu-romantischen Schule beschreibt. ${ }^{4}$ Die Armut von künstlerischen Erlebnissen war Anlass für ihr Komponieren. So lässt sich die kontextuale Ausgangslage für ihr Klaviertrio umreißen.

2 Hermann Krigar, "Konzerte”, in: Monatsschrift für Dramatik, Theater, Musik (März 1847), S. $47 f .$, hier S. 48.

3 Flodoard Geyer, "Wiederum acht neue Kammermusikwerke”, in: Neue Berliner Musikzeitung (17. November 1847), S. 381-385, hier S. 384.

4 Nancy Reich, Clara Schumann: The Artist and the Woman, revidierte Ausgabe (eBook), Ithaca 2013, hier insbes. Kapitel 11, "Clara Schumann as Composer and Editor". 


\section{Clara Schumann und die Sonatenform}

Clara Schumanns Verständnis der Sonatenform ist grundlegend für die Interpretation ihres Klaviertrios. Obwohl nur wenige Sonatenformsätze überliefert sind, ist ihre meisterhafte Anwendung im Trio eindeutig erkennbar. In 1842 hatte das junge Ehepaar die Streichquartette von Haydn und Mozart studiert, und als Interpretin kannte Clara natürlich Sonatenformen aller Art, vor allem viele Werke von Ludwig van Beethoven, Felix Mendelssohn und natürlich Robert. Zwei Stellen im Autographen sind sowohl Beispiele ihres klassischen Formbegriffs als auch Zeichen einer kalkulierenden, romantischen Künstlerin. In den Ecksätzen nummerierte sie Takte des Hauptsatzes, um zu Beginn der Reprise dann die wörtliche Wiederholung nur mit Taktzahlen zu notieren. Wie wir später in meiner Analyse sehen werden, sind diese einfachen Wiederholungen wichtige Bausteine einer satzübergreifenden, breiteren Struktur.

Während es nur wenige Sonatenformsätze von Clara Schumann gibt, ist die Situation für Robert anders. Seine Kreativität und Vorstellungskraft kommen in seinen vielen Sonataformsätzen zum vollen Ausdruck. Obwohl man sicherlich äußerst vorsichtig sein muss, Roberts Stil auf Clara zu beziehen, kann man wohl doch davon ausgehen, dass gemeinsames Studieren und ihr Interesse an Roberts Musik ihren subtilen Formbegriff mitgeprägt haben. Joel Lesters Aufsatz, "Robert Schumann and Sonata Forms", beschreibt die Form als "fertile ground in which he created a range of structurally compelling and aesthecially cogent relationships between thematic, tonal and other processes" . Lester schreibt weiterhin, dass "each of Schumann's sonata-form movements is individual in conception, because each uniquely relates its largescale structural and narrative plans to its thematic content ${ }^{\prime \prime}$. Clara Schumanns Op. 17 muss in diesem Sinn als ebenso kreativ und individuell interpretiert werden, denn die Komponistin nutzt diese Konventionen, um Inhalte zu vermitteln.

\section{Die zyklischen Zusammenhänge von Op. 17}

Satzübergreifende motivische Verbindungen sind allgemein bekannt, wie Joan Chissell und vor allem Janina Klassen sie schon beschrieben haben. Meine Interpretation basiert auf Klassens detaillierter Analyse. ${ }^{7}$ Dass motivische Zusammenhänge weit über die letzten beiden Sätze hinausgehen, sieht man am Beispiel des Trios des zweiten Satzes. Das Hauptthema des Andante wird schon in ähnlicher Form in dem Nachsatz des Trios vorgestellt (vgl. Notenbeispiele 1 und 3).

\footnotetext{
5 Joel Lester, „Robert Schumann and Sonata Forms”, in: 19th-Century Music 18, Nr. 3 (1995), S. 190.

6 Ebd., S. 190.

7 Janina Klassen, Clara Wieck-Schumann: die Virtuosin als Komponistin: Studien zu ihrem Werk (= Kieler Schriften zur Musikwissenschaft, Bd. 37), Kassel 1990.
} 


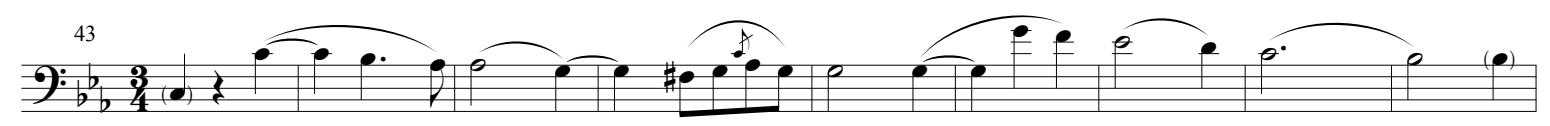

Notenbeispiel 3: Clara Schumann, Op. 17, zweiter Satz, Nachsatz des Trios

Das Autograph beinhaltet eine Skizzierung des Andante-Themas, möglicherweise als zweites Trio, was darauf hindeutet, dass Schumann die motivischen Zusammenhänge suchte (Abbildung 1).

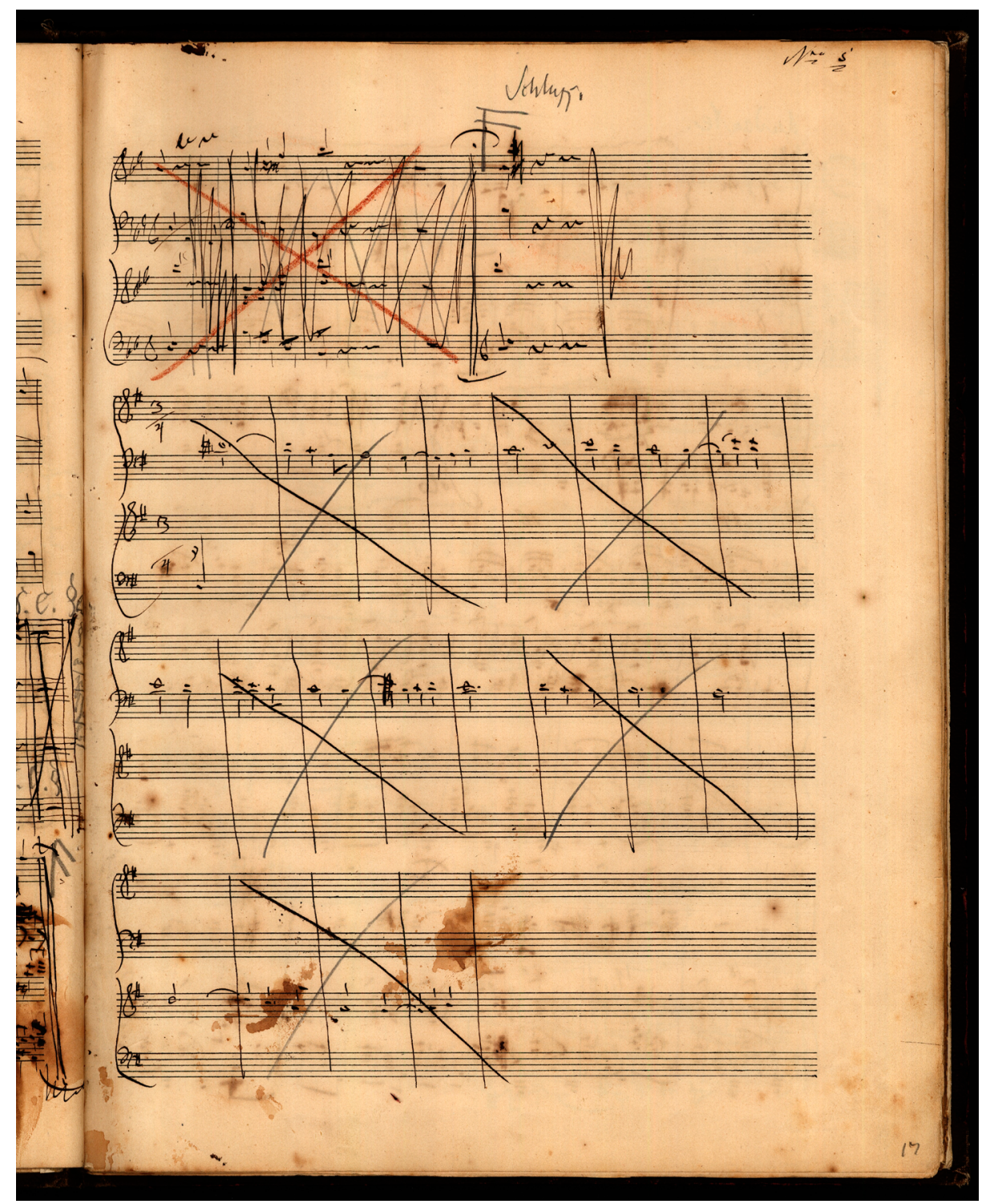

Abbildung 1: Clara Schumann, Op. 17, Autograph, Ende des zweiten Satzes 
Die diastematischen Elemente der zwei sequenziert fallenden Quinten sind aber auch beim Hauptthema des ersten Satzes präsent (Notenbeispiel 4). Noch deutlicher ist die fallende Quinte im Seitenthema des ersten Satzes zu hören. Obwohl das Klavier am Grundton vorbeiläuft, fixieren doch beide Streicher den Grundton als Endziel (Notenbeispiel 5). Anders als bei den anderen Themen wird jetzt die Quinte umgekehrt eine Sekunde tiefer sequenziert.

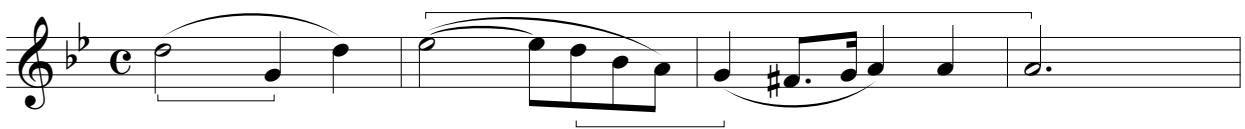

Notenbeispiel 4: Clara Schumann, Op. 17, erster Satz, Hauptthema

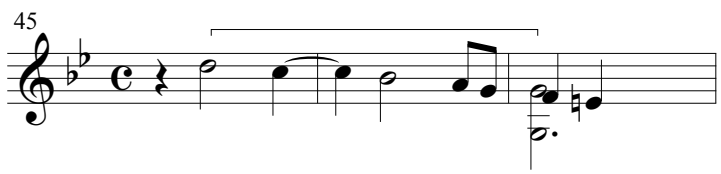

Notenbeispiel 5: Clara Schumann, Op. 17, erster Satz, Seitenthema

Nancy Reich identifiziert die fallende Quinte als ein "Clara-Motiv", das von beiden mehrfach in Kompositionen eingesetzt wurde (Notenbeispiel 6). ${ }^{8}$ In Roberts Sonate Op. 14 wird das "Clara Motiv" sogar benannt; und die Sonate beginnt auch mit diesem Motiv (Notenbeispiel 7).

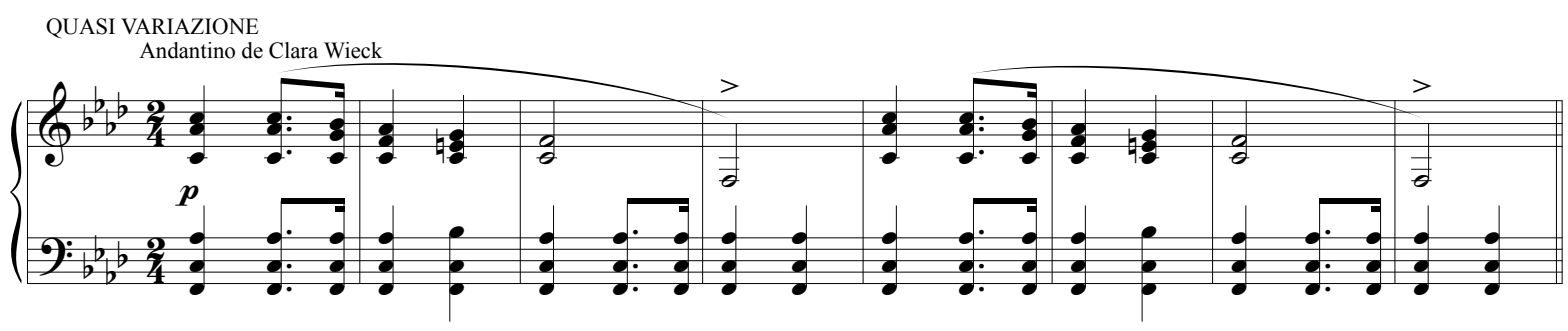

Notenbeispiel 6: Robert Schumann, Op. 14, dritter Satz

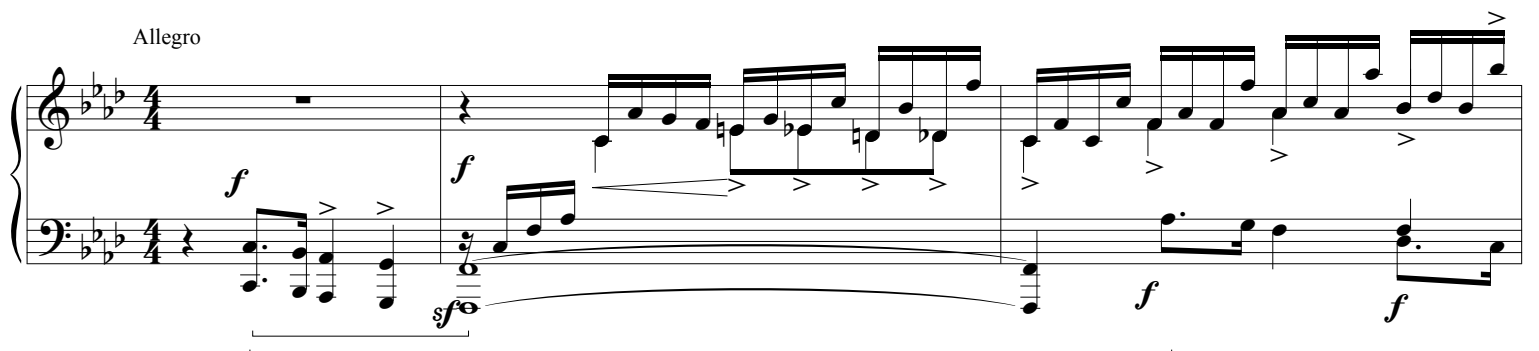

Notenbeispiel 7: Robert Schumann, Op. 14, erster Satz

8 Reich, Clara Schumann, Kapitel 11. 
Interessanterweise ist das Motiv ebenfalls in dem Hauptsatz von Roberts Klavierkonzert zu finden, und zwar gleich nach der fallenden Terz, die Claras Namen musikalisch buchstabiert (Notenbeispiel 8). Deutlicher ist dann die fallende Quinte in den Violinen zu hören, die dem oberen Nachbarton (Sechste) folgt. Es ist sicherlich kein Zufall, dass Clara in ihrem Klaviertrio eben dieses Motiv der Quinte mit Nachbarton in den Anfang mit einbezieht (Notenbeispiel 4). Hatte die Pianistin doch gerade vor Beginn ihrer Komposition das Klavierkonzert in Dresden und Leipzig uraufgeführt.

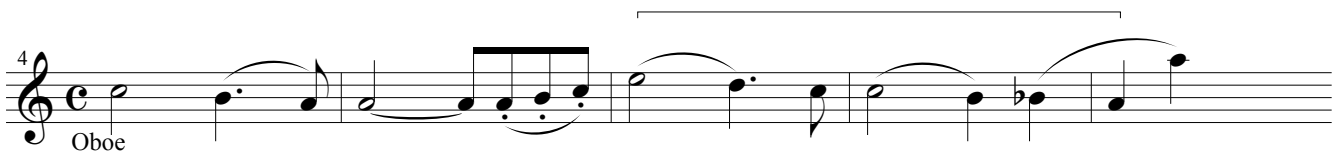

Notenbeispiel 8a: Robert Schumann, Op. 54, Hauptthema

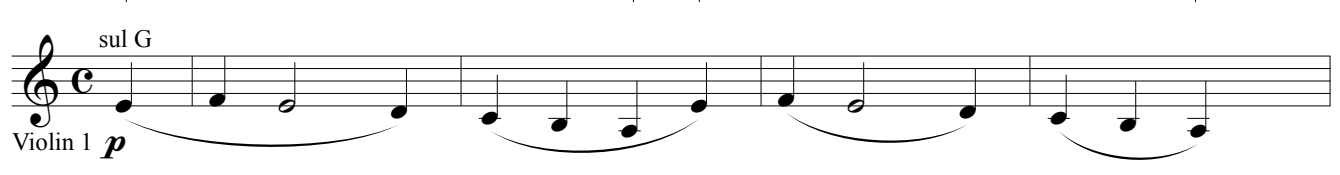

Notenbeispiel 8b: Robert Schumann, Op. 54, Hauptthema (Begleitung Takte 4-7)

Auch im zweiten Satz, dem Scherzo, erscheint die Quinte, jetzt in der Umkehrung (Notenbeispiel 9). Obwohl eingeflochten in das Thema, ist Claras Tenuto-Akzentuierung und LegatoBogen doch recht eindeutig in der Bestimmtheit dieses Motivs. Alle Sätze sind motivisch eng miteinander verbunden.

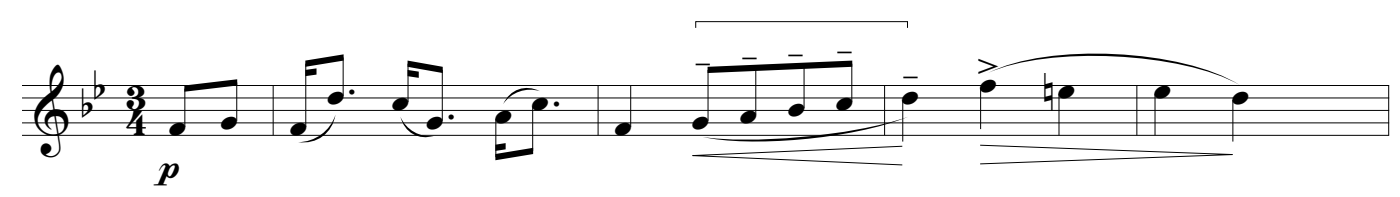

Notenbeispiel 9: Clara Schumann, Op. 17, zweiter Satz, Hauptthema

\section{„Fugenpassion“ und Roberts „Neue Art"}

"Ich habe das Meiste, fast alles, das kleinste meiner Stücke in Inspiration geschrieben, vieles in unglaublicher Schnelligkeit, so meine 1ste Symphonie in B-Dur in vier Tagen, einen Liederkreis von zwanzig Stücken ebenso, die Peri in (ebenso) verhältnismäßig ebenso kurzer Zeit. Erst vom Jr. 1845 an von wo ich anfing alles im Kopf zu erfinden und auszuarbeiten, hat sich eine ganz andere Art zu componiren zu entwickeln begonnen". 9

9 Robert Schumann, Tagebücher Band 2 (1836-1854), hrsg. von Gerhard Nauhaus, Frankfurt a.M. 1987, S. 402. 
Ein Lichtblick in dem verhängnisvollen Umzug nach Dresden war die nun überraschende Möglichkeit, sich ganz auf das Komponieren konzentrieren zu können. Mit der Geburt von Tochter Julie am 8. März war Clara nicht in der Lage ans Konzertieren zu denken. Das Ehepaar entschied sich also noch einmal Kontrapunkt zu studieren. Diesmal arbeiteten sie Cherubinis Theorie des Contrapunktes und der Fuge ${ }^{10}$ durch. Wie schon vor einigen Jahren analysierten sie auch Präludien und Fugen von Bachs Wohltemperiertem Klavier. Der Eifer und Erfolg dieser Studie führte u. a. zur Veröffentlichung von Roberts Sechs Fugen über den Namen "Bach" und Claras Drei Präludien und Fugen Op. 16. Wichtiger allerdings ist wie sehr das Kontrapunkt-Studium Roberts Stil im Allgemeinen geprägt hat. John Daverio beschreibt, wie zum Beispiel seine Charakterstücke davon beeinflusst wurden. ${ }^{11}$ So führte dann nach Roberts eigener Aussage die Fugenpassion zu einer neuen Methode.

Claras "moderner" Ansatz ist am klarsten in ihrem dritten Paar zu erkennen. Hier sind Präludium und Fuge thematisch eng miteinander verbunden. Daverio nennt Roberts neuen Stil Ars combinatoria; das kompositorische Material ist nicht mehr die Melodie, sondern eine Vergliederung von Motiven. ${ }^{12}$

Dass Clara wohl eine ähnliche Richtung einschlug, ist an dem Hauptthema des ersten Satzes leicht erkennbar (Notenbeispiel 10). Während die fallende Quinte in der Violine in das Thema subtil eingebunden ist, wird die fallende Quinte im Bass die Basis auf dem das Thema beruht (wie in Roberts Klavierkonzert). Ebenso findet sich im Seitenthema Claras Ars combinatoria. Der Vordersatz mit der fallenden Quinte und der Nachsatz mit verminderten Septakkorden und chromatischer Achtelfigurierung werden in der Wiederholung des Themas kontrapunktisch gegenübergestellt (Notenbeispiele 11a und 11b). Geyer empfand diese Stelle besonders bemerkenswert, wenn er das "Gegenthema des zweiten Themas" in der Neuen Berliner Musikzeitung als Beispiel für die "Beherrschung der formellen Kunstmittel" hervorhebt. ${ }^{13}$

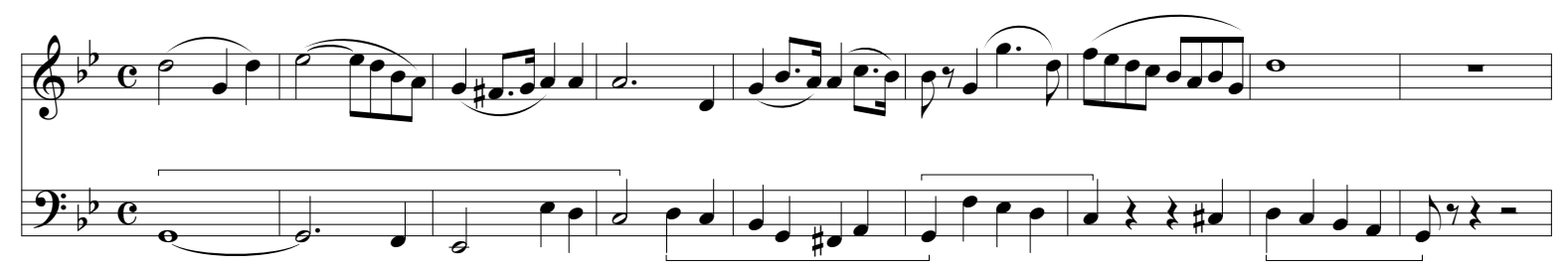

Notenbeispiel 10: Clara Schumann, Op. 17, erster Satz, Hauptthema

10 Luigi Cherubini, Theorie des Contrapunktes und der Fuge, aus dem Französchen von Frantz Stoepel, Leipzig, 1834.

11 John Daverio, Robert Schumann: Herald of a "New Poetic Age", New York, 1997, S. 308-310.

12 Ebd., S. 322-325.

13 Geyer, "Wiederum acht neue Kammermusikwerke", S. 384. 

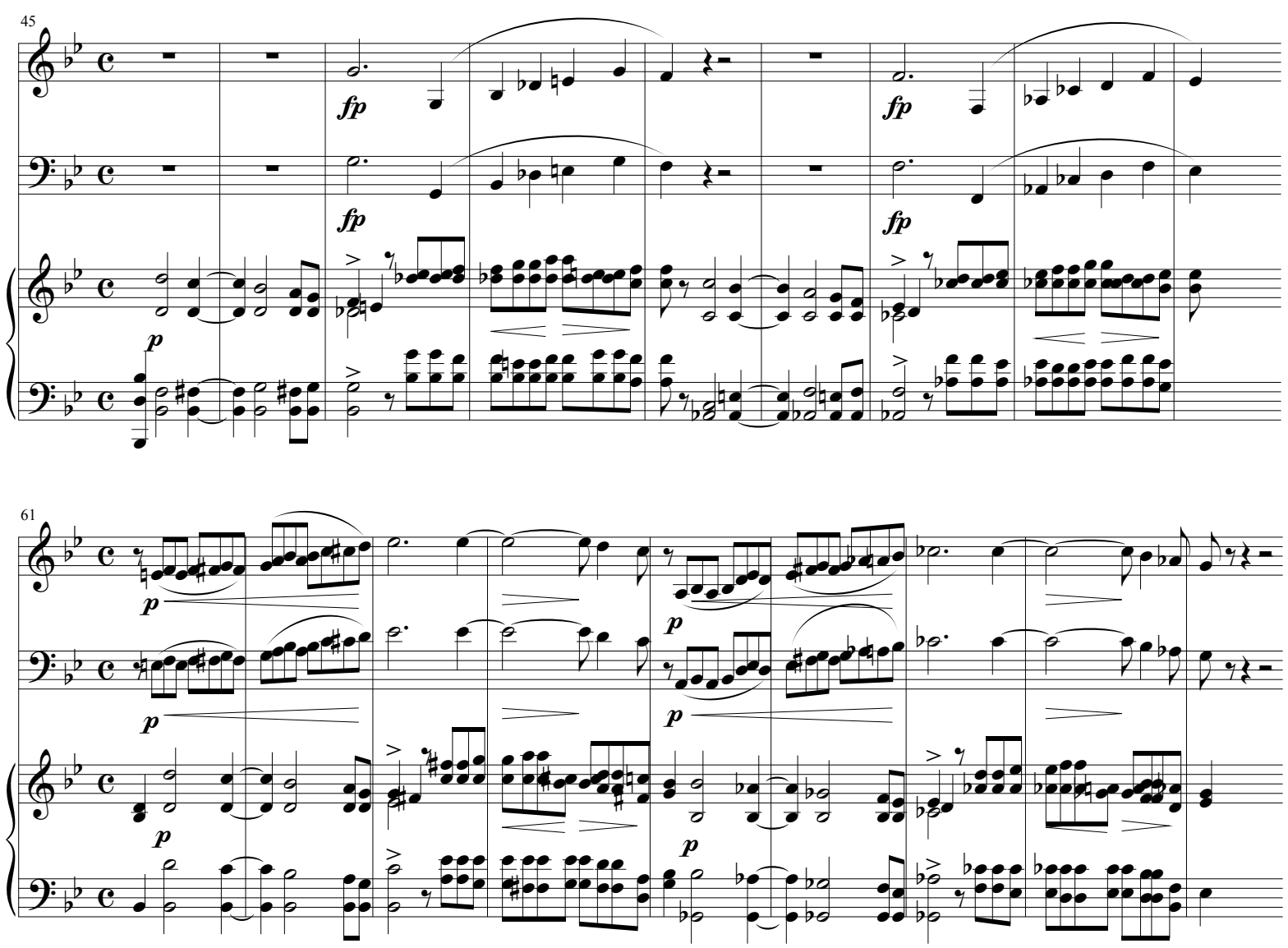

Notenbeispiele 11a-b: Clara Schumann, Op. 17, erster Satz, Seitenthema

\section{Sonatenformstrukturen}

Janina Klassens Analyse des ersten Satzes zeigt, dass Haupt- und Seitenthema wegen organischer Verknüpfung ungewöhnlich wenig Kontrast bieten. ${ }^{14}$ Die Durchführung verarbeitet Motive wie den Themenkopf des Hauptthemas durch kontrapunktische Zerlegung sowie durch Kombination mit dem Seitensatz. Anstatt Synthese steht Verknüpfung weiter im Vordergrund. Die Reprise wiederholt wie schon vorher erwähnt wortwörtlich das erste Thema. Hier ist wiederum keine Synthese, Weiterentwicklung oder Variation zu finden. Synthese findet nur harmonisch statt, denn wie erwartet erscheint das Seitenthema nun in der Tonika. Die Coda offeriert dann letztendlich nur eine Steigerung durch ein schnelleres Tempo. Am Ende des ersten Satzes ist der Hörer in einer ungewohnten Lage. Weder Kontrast noch Synthese, Tragik, oder irgendeine andere dynamische Entwicklung wurden bis jetzt vorgestellt. Ein aufatmendes Charakterstück

14 Klassen, Clara Wieck-Schumann, S. 214f. 
oder eine poetische Aufheiterung sind nicht notwendig, denn bis jetzt hörte man nur ähnliche Themenkreise und deren Verarbeitung. So ist das Scherzo dann nicht, wie erwartet in G-Dur oder g-Moll und auch nicht in Es-Dur, die Tonart eines langsamen Satzes, sondern in B-Dur, der Tonart des Seitenthemas.

Und das Scherzo ist auch nicht wirklich ein Scherzo, wie die Revision "Tempo di Menuetto" aufzeigt. Der Lombardische Rhythmus, oder "Scotch snap", und die Sprünge von Sexte, Quarte, und Terz bilden einen Kontrast zum ersten Satz. Das Trio und das darauffolgende variierte Scherzo geben dem zweiten Satz die Funktion eines Charakterstücks in dreiteiliger Form. Die Allgemeine musikalische Zeitung erkennt in dem Scherzo ein "neckisches, kindlich fröhliches Wesen", das im Kontrast zu dem "Charakter [...] von leiser Wehmut" steht. ${ }^{15}$ Zusammengenommen könnte man die beiden ersten Sätze als eine Exposition auffassen.

Mit dem schon erwähnten motivischen Zusammenhang von Trio und Andante wird der Hörer nun nahtlos in den nächsten Satz geführt. Wichtiger jedoch als einfacher motivischer Zusammenhang ist wie das Andante-Thema die diastematischen Eigenschaften des ersten Satzes aufgreift und verwandelt: Die fallende Quinte wird von Moll nach Dur verwandelt, die verminderte Quinte Es-A wird zu E-A aufgelöst, und sogar die synkopisch fallende Quinte des Seitenthemas wird im Verlauf des Themas aufgegriffen. In der nächsten Phrase spielt dann die Geige eine Erweiterung des Nachsatzes, während das Cello verminderte Quinten sequenziert (Notenbeispiele 12a-b).

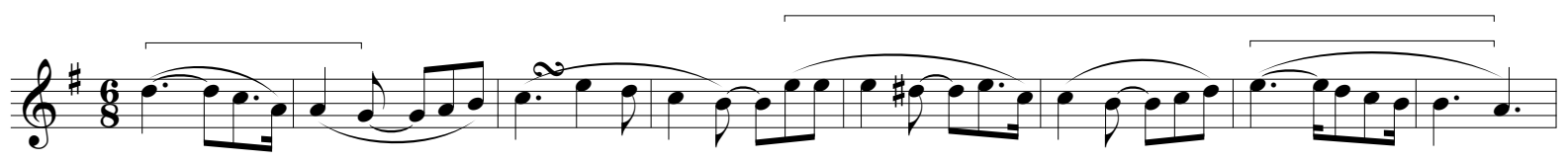

Notenbeispiele 12a: Clara Schumann, Op. 17, Dritter Satz, Hauptthema

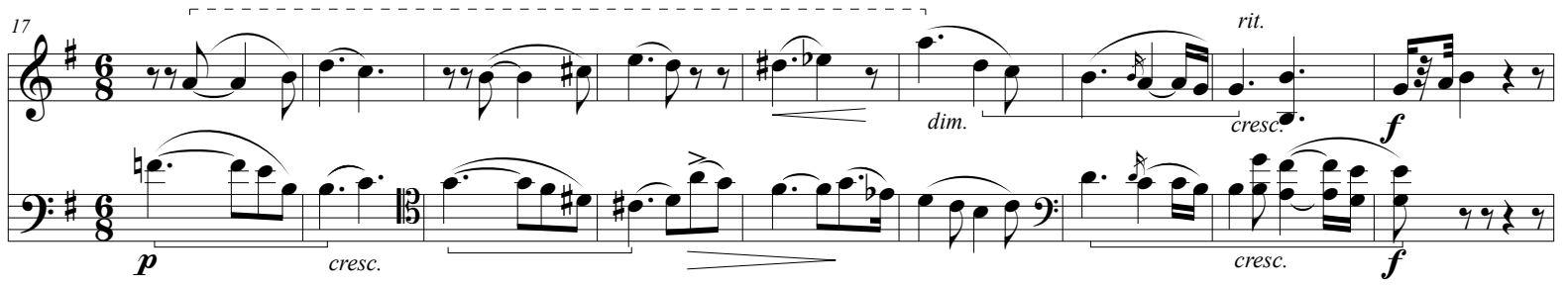

Notenbeispiele 12b: Clara Schumann, Op. 17, Dritter Satz, Hauptthema - kontrapunktische Erweiterung

Schaut man wieder auf die Rezensionen, so ist das Andante eine Insel von "Trost und Hoffnung", die von "stiller Trauer umflossen" ist. ${ }^{16}$ Die "einfachen Liedklänge" werden von dem Klavier solo

15 „Rezensionen“, Allgemeine musikalische Zeitung (5. April 1848), S. 233.

16 Geyer, „Wiederum acht neue Kammermusikwerke”, S. 384. 
vorgestellt. ${ }^{17}$ Es ist einer von wenigen Augenblicken, in denen Schumann das Klavier in den Mittelpunkt rückt, was das Andante umso persönlicher macht. Im Autograph spielte die Geige von Anfang an das Thema. Erst in der revidierten Fassung werden die ersten acht Solo-Klavier Takte vorgeschoben, was den Typus des Charakterstücks unterstreicht.

In die heile Welt in G-Dur bricht ein turbulenter e-Moll Mittelteil, der die Idylle mit doppelt punktierten Rhythmen zerschlägt. Nachdem verbindende motivische Elemente der Haupt- und Seitenthemen im A-Teil aufgegriffen wurden, bearbeitet der Mittelteil jetzt die Motivik der Überleitung aus dem ersten Satz. Dabei greift das Thema des Mittelteils auch die Punktierung und die Sext-, Quart-, und Terzsprünge des Scherzos auf. Klassen beschreibt den Mittelteil als einen Kulminationspunkt des Werkes im Scherzo-Stil. ${ }^{18}$ Allerdings sind wir tonartlich mit e-Moll weit entfernt von der Tonika des Werkes, das heißt harmonisch hat die Kulmination eher die Merkmale einer Durchführung. Trost und Hoffnung werden hier in Frage gestellt. Dennoch endet das Andante ruhig in G-Dur, und das Clara-Motiv aus Haupt- und Seitenthema des ersten Satzes rückt in der Coda wieder in den Vordergrund.

Wie schon vorher erwähnt, der Anfang des letzten Satzes ist überraschend, wenn nicht sogar schockierend. Das zunächst idyllisch erscheinende Andante wurde durch den Mittelteil in Frage gestellt, und nun bricht die Idylle mit einer thematischen Metamorphose völlig zusammen, die uns wieder nach g-Moll reißt - und die diastematischen Merkmale des ersten Satzes sind erneut präsent (Notenbeispiele 13). Wir sind allerdings nicht wieder einfach am Anfang, denn der Charakter der fallenden Quinte hat sich durch die Chromatik der oberen Terz zugespitzt, auf die, wie wir jetzt feststellen, schon im Scherzo angespielt wurde (Notenbeispiel 9, Takte 3-4). Auch treibt der Nachsatz melodisch nicht mehr aufwärts. Paradox erscheinen deshalb die Arpeggien im Klavier, die im völligen Kontrast zur Melodie stehen. Lamento und Trost scheinen sich gegenüber zu stehen. Und es ist diese paradox anmutende Resolution, die sich nun wie ein roter Faden durch das Finale zieht.

Die Reprise von diastematischen Merkmalen setzt sich in dem Seitenthema fort. Überraschenderweise in D-Dur anstatt B-Dur werden nun zunächst sowohl die Punktierung und steigende Quinte des Scherzos als auch das Seitenthema des ersten Satzes rekapituliert. Der schrittweise steigenden Quinte steht die fallende Quinte in den Streichinstrumenten gegenüber. In der nächsten Periode hören wir dann eine Umkehrung der Septime (Notenbeispiele 5 und 14a). In der Phrasenwiederholung spielt die Geige eine Sexte als Kontrapunkt mit einem Einschub der Quinte des Cellos (Notenbeispiel 14b). Die rekapitulatorischen Faktoren in Claras Arscombinatoria-Stil werden durch den Scherzo-Charakter des Seitenthemas untermauert. Der eben genannte Sextsprung wird nun im Nachsatz mehrmals aufgegriffen, und resümiert auch im Zusammenhang des Scherzo-Charakters die markante Sexte des zweiten Satzes.

17 Ebd.

18 Klassen, Clara Wieck-Schumann, S. 246. 

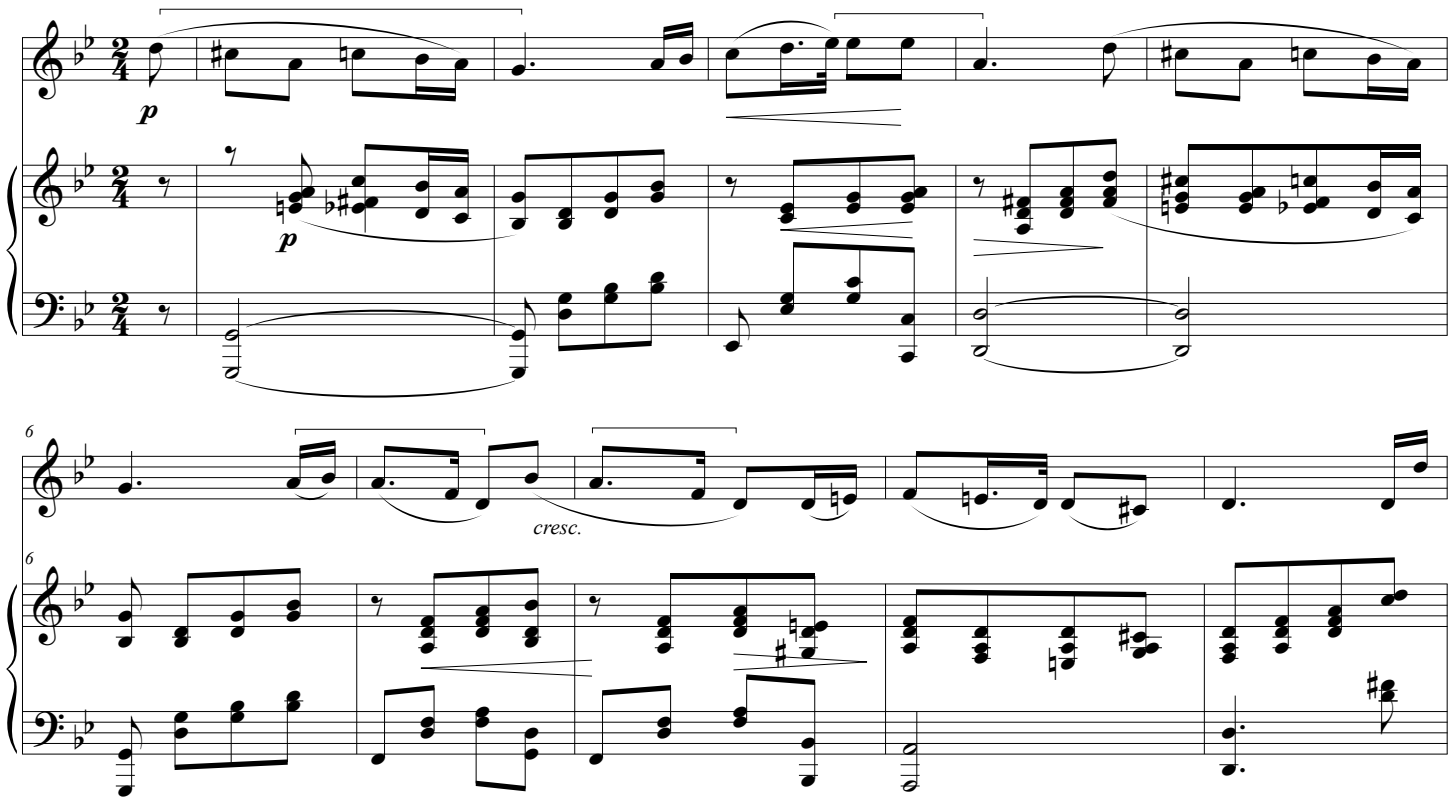

Notenbeispiel 13: Clara Schumann, Op. 17, Vierter Satz, Hauptthema

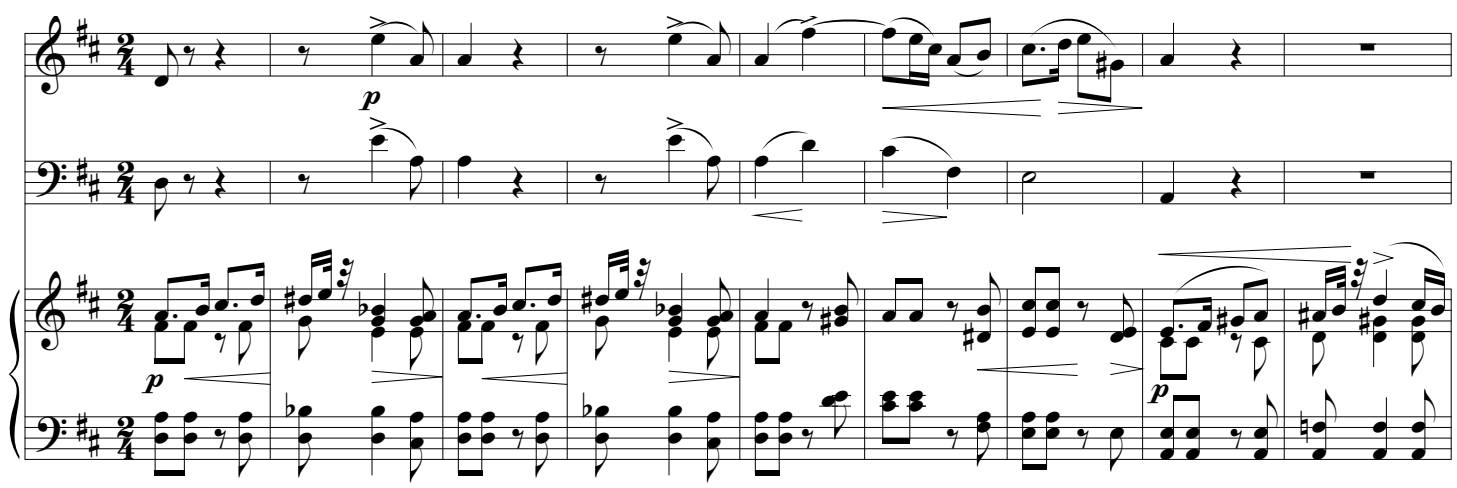

Notenbeispiele 14a: Clara Schumann, Op 17, vierter Satz, Seitenthema, Takt 56-64

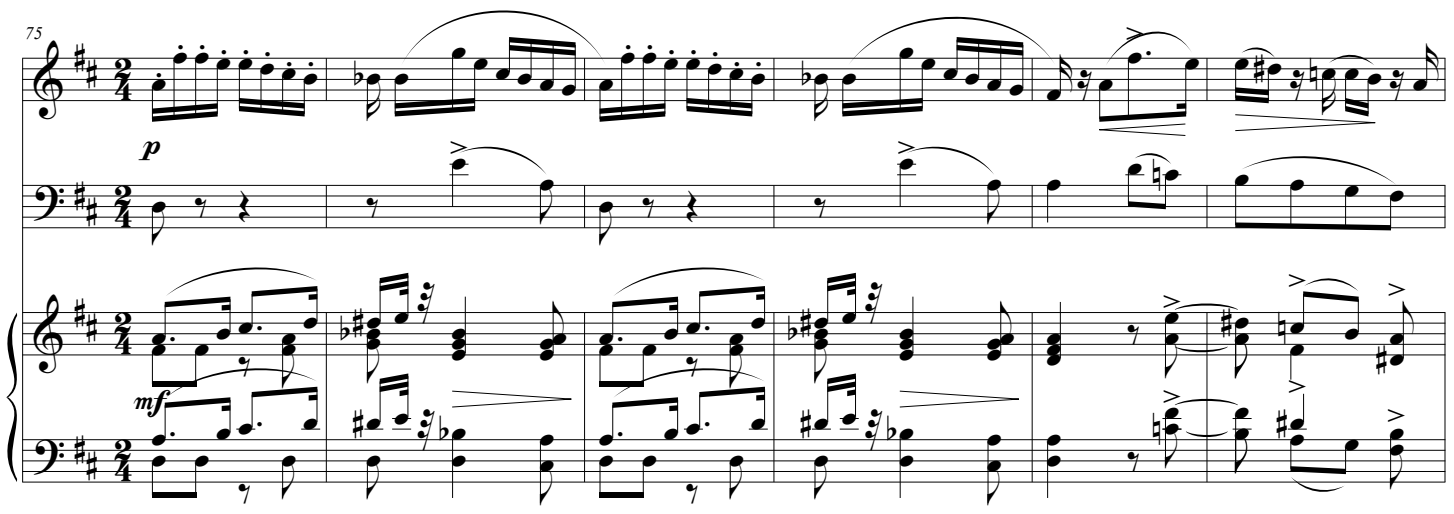

Notenbeispiele 14b: Clara Schumann, Op 17, vierter Satz, Seitenthema, Takt 75-80 
Die auffälligste Besonderheit des Finales ist natürlich das Fugato. Das Thema ist eine Adaption des Hauptsatzes. Mit dem dritten Einsatz im Cello spielt das Klavier eine chromatisch fallende Quinte - eine Destillation des Themas (Notenbeispiel 15). Das Fugato wird mit Einschüben des Seitensatzes unterbrochen, die mit einer steigenden Quinte und punktierten Rhythmen genau das Gegenteil darbieten. Die dramatische Durchführung zeigt, dass die sich andeutende Reprise in der Exposition des letzten Satzes keine anhaltende Auflösung offeriert.

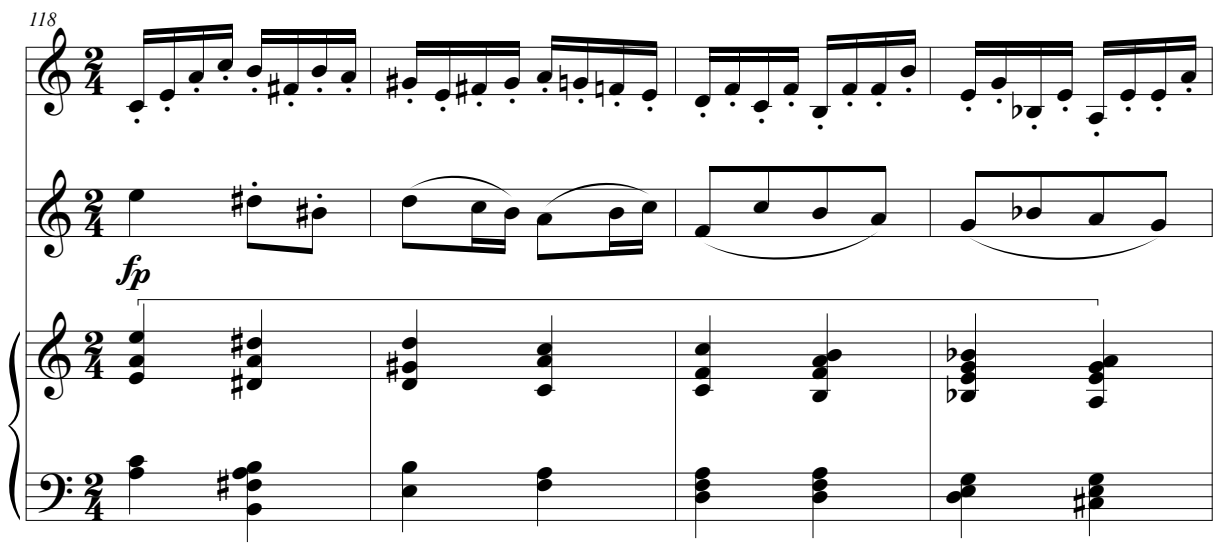

Notenbeispiel 15: Clara Schumann, Op. 17, vierter Satz, Fugato

Das Paradox des letzten Satzes setzt sich mit Überleitung und Beginn der Reprise fort. Die Rückkehr des Hauptthemas ist alles andere als der Höhepunkt. Es ist wieder einige der wenigen Stellen, wo das Klavier total in den Vordergrund rückt. Jedoch hört sich der sich reinschleichende Hauptsatz wie Resignation an. Und nur mit dem Einsatz der Geige kommt die Komposition endlich wieder in Gang.

Das Paradox spitzt sich mit dem Seitenthema zu, denn die Tonart ist jetzt B-Dur. Nichts hat sich entspannt, weder thematisch noch harmonisch. Sieht man die ersten beiden Sätze als Exposition, so offeriert der letzte Satz zwar eine Reprise der diastematischen Besonderheiten, aber keine konventionelle Aufhebung der harmonischen Spannung, sondern eher eine Zuspitzung.

Aber selbst die Coda bringt keine Resolution. Die erste Fassung endet sogar auf dramatische Weise in Moll (Abbildung 2). In der Druckfassung wird ein Ende in G-Dur herbei beschwört. Es ist alles andere als befriedigend. Interessanterweise ist das neue Ende dem Schluss des dritten Satzes - dem Satz von Trost und Hoffnung - sehr ähnlich. 


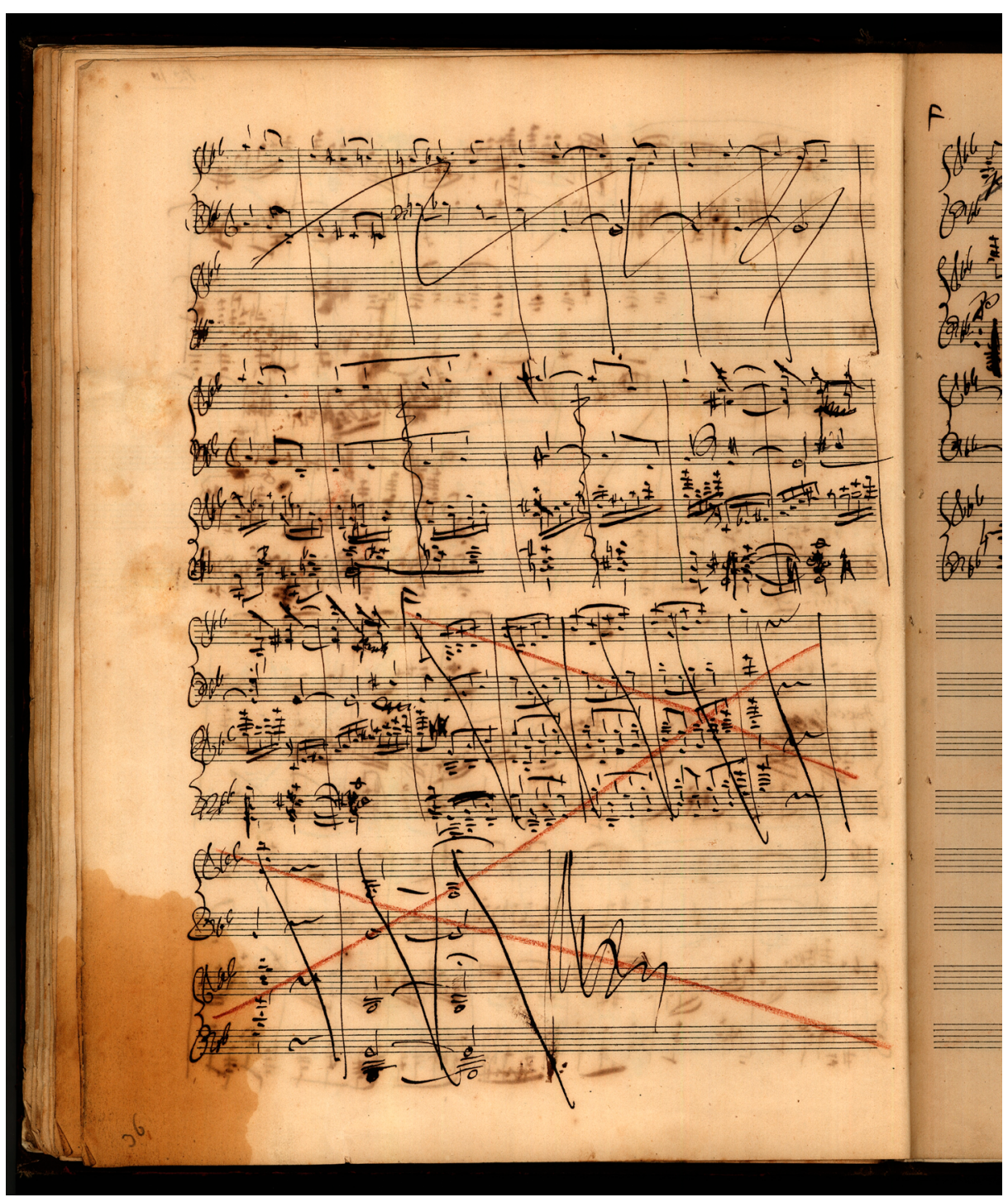

Abbildung 2: Clara Schumann, Op. 17, Autograph, Ende des vierten Satzes (verworfene Fassung)

In den Rezensionen wird dem letzten Satz große Aufmerksamkeit geschenkt. Die Allgemeine musikalische Zeitung beschreibt die harmonischen Anomalien dieses Sonatensatzes, ohne sie zu tadeln. Im Gegenteil, die Musik hat dadurch Aussagekraft, die durch den "melodischen Fluss" ein Ganzes schafft. ${ }^{19}$ Und die Neue Berliner Musikzeitung resümiert, dass "der letzte Satz" eine "durchweg [...] meisterhafte Anlage" hat. ${ }^{20}$ Das Finale ist nicht der Höhepunkt eines Geschehens. Es ist vielmehr ein Ausdruck des trotzenden Daseins. Diese gegensätzlichen Ereignisse - oder vielleicht besser Empfindungen - der ersten Sätze führen nicht zu einer Lösung. Die Gegensätze sind und bleiben vorhanden, und sie werden mit Entschlossenheit ertragen.

19 „Rezensionen“, $A m Z$ (5. April 1848), S. 233.

20 Geyer, "Wiederum acht neue Kammermusikwerke", S. 384. 


\section{Fazit}

Clara Schumann war 1846 eine erfahrene Künstlerin. Sie hatte schon viel von der Welt gesehen. Sie hatte sich gegen ihren Vater gestellt, Robert fortan unterstützt und war Mutter geworden. Als sie ihre Heimat Leipzig verließ, musste sie endgültig ihre Jugendzeit zurücklassen. Diesmal konnte sie der Situation auch nicht mit Konzertreisen entfliehen. Ihren schwierigen und frustrierenden Erlebnissen stellte sie sich mit Entschlossenheit - ein Wesenszug, der sie für den Rest ihres Lebens auszeichnete. Das Klaviertrio gab ihr die Möglichkeit sich dennoch künstlerisch auszudrücken, und sie nutzte alle ihr zur Verfügung stehenden kompositorischen Mittel, ihrer Frustration und ihrer Entschlossenheit Ausdruck zu verleihen.

Zitation: Siegwart Reichwald, „Die Leiden der jungen Clara: Das Klaviertrio Opus 17 als Ausdruck einer Neu-Romantikerin", in: Freie Beiträge zur Jahrestagung der Gesellschaft für Musikforschung 2019, hrsg. von Nina Jaeschke und Rebecca Grotjahn (= Musikwissenschaft: Aktuelle Perspektiven. Bericht über die Jahrestagung der Gesellschaft für Musikforschung 2019 in Paderborn und Detmold, Bd. 1), Detmold 2020, S. 277-291, DOI: 10.25366/2020.69. 


\section{Abstract}

Two reasons precipitated the move of the Schumanns to Dresden: Robert Schumann's health and the reconciliation with Clara Schumann's father. Yet Dresden quickly turned into disappointment: Robert's mental condition worsened, relations with her father deteriorated. Burdened with familial responsibilities and no time for concert tours, Clara found expression in her most ambitious and melancholic composition, creating one of the most individualistic and innovative chamber works of her generation. Central to this narratological reading is the composer's use of the Clara theme (diatonically descending fifth) and its many derivatives throughout the work. Substantial revisions found in the autograph (RSH 12897-A1) present obvious clues about overarching thematic and harmonic strategies and early reviews emphasizing the composer's individualistic voice offer further hermeneutic insights. To express her disappointing Dresden experience defiantly, Clara explored the possibilities of thematic integration among all four movements as well as a super-imposed sonata form design across the whole work.

\section{Kurzvita}

Siegwart Reichwald wurde 1967 in Hameln geboren. Nach seinen Bachelor an der University of South Carolina, Columbia im Fach Orgel absolvierte er einen M. M. im Fach Dirigieren. Seine Dissertation, mit dem Titel The Musical Genesis of Felix Mendelssohn's Paulus erfolgte an der Florida State University, Tallahassee. 


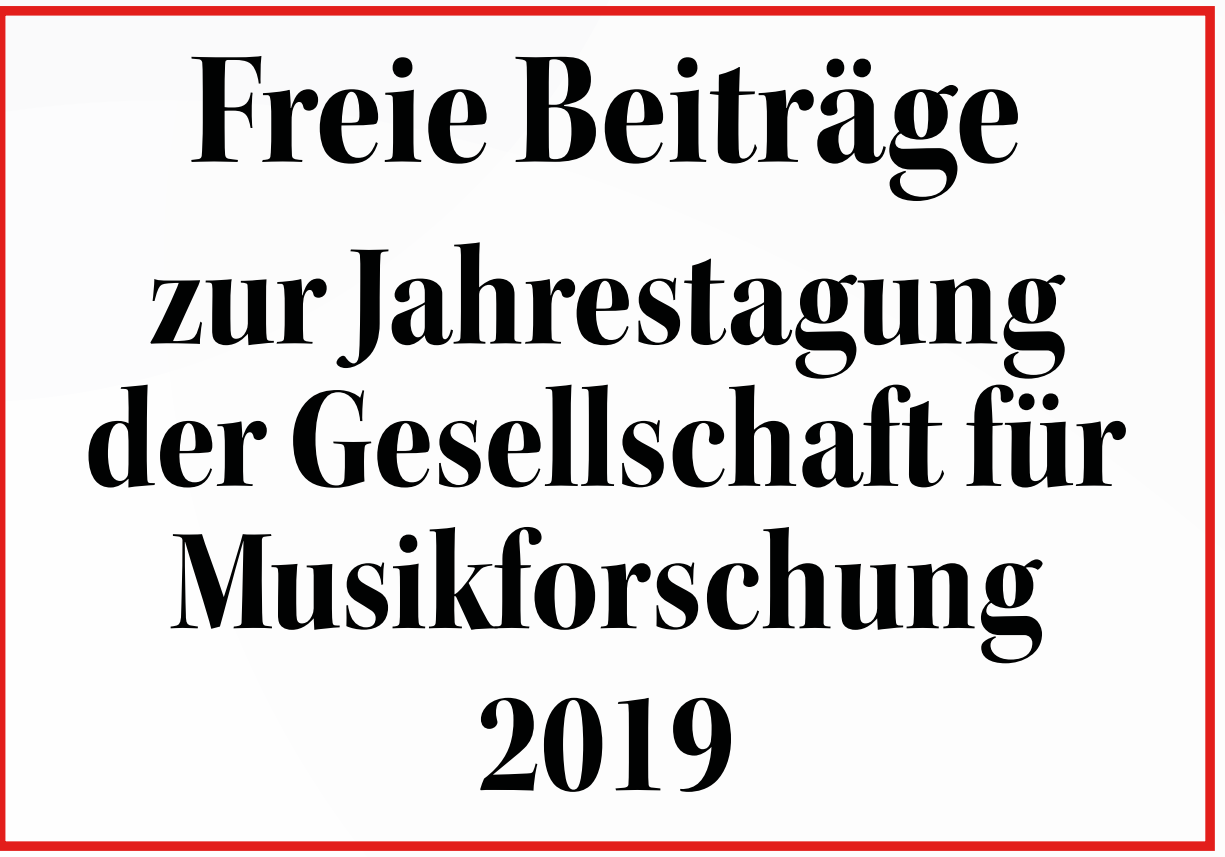

Herausgegeben von Nina Jaeschke und Rebecca Grotjahn

Musikwissenschaft: Aktuelle Perspektiven 1 
Freie Beiträge 


\section{Musikwissenschaft: Aktuelle Perspektiven}

Bericht über die Jahrestagung der Gesellschaft für Musikforschung 2019 in Paderborn und Detmold

Herausgegeben von Rebecca Grotjahn und Nina Jaeschke

Band 1 


\section{Freie Beiträge}

\section{zur Jahrestagung der Gesellschaft für Musikforschung 2019}

Herausgegeben von Nina Jaeschke und Rebecca Grotjahn

Detmold: Musikwissenschaftliches Seminar der Universität Paderborn und der Hochschule für Musik Detmold 2020 
DOI: $10.25366 / 2020.42$

Online-Version verfügbar unter der Lizenz: Urheberrecht 1.0, $<$ https://rightsstatements.org/page/InC/1.0/?language=de>

Bibliografische Information der Deutschen Nationalbibliothek

Die Deutsche Nationalbibliothek verzeichnet diese Publikation in der Deutschen Nationalbibliografie; detaillierte bibliografische Daten sind im Internet über http://dnb.d-nb.de abrufbar.

\section{Impressum}

Redaktion: Nina Jaeschke, Rebecca Grotjahn und Jonas Spieker Satz: Nina Jaeschke

(C) Musikwissenschaftliches Seminar der Universität Paderborn und der Hochschule für Musik Detmold 2020 


\section{INHALT}

Vorwort $\quad$ IX

Komponieren für das Radio: Akteure, Diskurse, Praktiken $\quad 1$

Musikwissenschaft - Feminismus - Kritik: Ein Generationenaustausch 6

\section{Stefan Alschner}

Der Wagner-Sänger Joseph Aloys Tichatschek - Vom Nachlass zum Netzwerk

\section{Alenka Barber-Kersovan}

Songs for the Goddess. Das popmusikalische Neo-Matriarchat zwischen Ethno-Beat,

erfundenen Traditionen und kommerzieller Vermarktung

Elias Berner, Julia Jaklin, Peter Provaznik, Matej Santi, Cornelia Szabó-Knotik

Musikgeschichte anders erzählen? Das Beispiel der 1970er in Österreich.

Musikhistoriographie in der Zeit der Digitalisierung

\section{Mauro Fosco Bertola}

„Ein Laut so klagevoll”. Lohengrin zwischen Richard Wagner und Salvatore Sciarrino

\section{Matthieu Cailliez}

Europäische Rezeption der Berliner Hofoper und Hofkapelle von 1842 bis 1849

\section{lacopo Cividini}

Zwischen klassischer Musikphilologie und angewandter Informatik:

Die Digitale Mozart-Edition (DME) der Stiftung Mozarteum Salzburg

\section{Marko Deisinger}

Fortschrittliche Technologie im Dienste eines Antimodernisten.

Heinrich Schenker und der österreichische Rundfunk

\section{Norbert Dubowy}

Vom Kritischen Bericht zur Kritischen Dokumentation am Beispiel der Digital-interaktiven Mozart-Edition

\section{Markus Engelhardt}

Musik zwischen Nation Building und Internationalität. Italien um 1900

\section{Maryam Haiawi}

Das Oratorium im Spannungsfeld der Konfessionen: 


\section{Judith I. Haug}

"Manch eine*r liegt, morgens noch trunken, im Rosengarten" - Rekonstruktionen

osmanischer Musikgeschichte in Gesangstextsammlungen

\section{Renate Koch}

Marcel Prawy und das erste Broadway-Musical im Österreich der Nachkriegszeit

Susanne Kogler, Julia Mair, Juliane Oberegger, Johanna Trummer

Erich Marckhl - Musikausbildung in der Steiermark nach 1945.

Brüche und Kontinuitäten

\section{Marie-Anne Kohl}

Die weinende Jury. "Geschlechtslose" Tränen bei globalen Musik-Castingshows?

\section{Fabian Kolb}

Tanztheater und filmische Ästhetik. Cineastische Einflüsse und Gestaltungsweisen in den Kompositionen für die Ballets Suédois 1920-1925

\section{Christian Lehmann}

Tempobezeichnungen von Julius Stockhausen für Die schöne Müllerin:

Ein Quellenfund

\section{Martin Link}

Signum et gens - Zur Gendersemiotik in Clara und Robert Schumanns Liederzyklus Liebesfrühling

\section{Livio Marcaletti}

„Strafspiel" und satirische Stilmittel in musikdramatischen Gattungen des frühen 18. Jahrhunderts

\section{Tobias Marx, Martin Lissner}

Thüringer Musikszene - Jugendmusikredaktionen als außerschulische musikbezogene Bildungskontexte

\section{Maho Naito}

Die Parallelität der Entstehungsprozesse der ersten beiden Symphonien Gustav

Mahlers: Instrumentation, Revision und Dirigierpraxis

\section{Elisa Novara}

Eine Schumann-Werkstatt? Zur Übertragbarkeit der Methoden vom Projekt 
Theodora Oancea, Joachim Pollmann, Jonas Spieker

Kollaborateure - Involvierte - Profiteure. Erarbeitung eines Online-Lexikons zur

Musik in der NS-Zeit

\section{Kiron Patka}

„Ich wollte eigentlich Sängerin werden." Berufsselbstbilder von Tontechniker*innen im Radio

\section{Siegwart Reichwald}

Die Leiden der jungen Clara: Das Klaviertrio Opus 17 als Ausdruck einer Neu-

Romantikerin

\section{Elisa Ringendahl}

Lied versus Oper - Pole musikalischer Gattungen bei Oscar Bie

\section{Benedikt Schubert}

Struktur und Exegese. Über Eigentümlichkeiten in der Arie "Des Vaters Stimme ließ sich hören" (BWV 7/4)

Uwe Seifert, Sebastian Klaßmann, Timo Varelmann, Nils Dahmen

Computational Thinking in der Musikwissenschaft: Jupyter Notebook als Umgebung

für Lehre und Forschung

\section{Yusuke Takamatsu}

Synthese als Modus der Prozessualität bei Schubert:

Sein spezifisches Wiederholungsprinzip im langsamen Satz

\section{Daniel Tiemeyer}

Johann Nepomuk Hummels Sonate in fis-Moll Op. 81 - Studien zu Entstehungs-

hintergrund, Rezeption und formalerStruktur

\section{Andrea van der Smissen}

Musikalische Innovation im Umfeld der Moderne und historischen Avantgarde in Ungarn

\section{Tim Ziemer, Holger Schultheis}

Psychoakustische Sonifikation zur Navigation in bildgeführter Chirurgie

\section{Magdalena Zorn}

Musik mit dem Radio hören: Über den Begriff der musikalischen Aufführung 
Gabriele Buschmeier in memoriam 


\section{Vorwort}

Die vorliegenden Bände dokumentieren die Jahrestagung der Gesellschaft für Musikforschung 2019. In den dreieinhalb Tagen vom 23. bis zum 26. September 2019 wurden in Paderborn und Detmold nicht weniger als 185 Beiträge präsentiert, verteilt auf diverse Symposien, Round tables, Freie Sektionen und Postersessions. Sie alle auf einen Nenner bringen zu wollen, ist ein Ding der Unmöglichkeit - und das ist gut so, ist es doch Ziel der Jahrestagungen, die große Vielfalt der Themen und Methoden des Faches Musikwissenschaft abzubilden. Um die thematische Vielfalt der freien Referate angemessen abbilden zu können und gleichzeitig den inhaltlichen Schwerpunkten der beiden hier publizierten Hauptsymposien ausreichend Raum bieten zu können, erscheinen diese in drei Bänden.

„Musikwissenschaft: Aktuelle Perspektiven": Der Titel der kleinen Reihe ist keine Verlegenheitslösung. Musikwissenschaft im Kontext der Digital Humanities; Musikwissenschaft und Feminismus; Musik und Medien; Musikalische Interpretation - schon die Felder, die von den vier Hauptsymposien bespielt wurden, wären noch vor wenigen Jahrzehnten allenfalls an der Peripherie das Faches zu finden gewesen. Sie entsprechen Arbeitsschwerpunkten der Lehrenden am Musikwissenschaftlichen Seminar der Universität Paderborn und der Hochschule für Musik Detmold, das die Tagung ausrichtete. Zugleich nehmen sie Bezug auf aktuelle Ereignisse und Entwicklungen. So erwuchs das von Andreas Münzmay und Joachim Veit organisierte Symposium „Brückenschläge - Informatik und Musikwissenschaft im Dialog" unmittelbar aus den Erfahrungen im Virtuellen Forschungsverbund Edirom (ViFE) und im fakultäten- und hochschulübergreifenden Zentrum Musik-Edition-Medien (ZenMEM). Der 200. Geburtstag von Clara Wieck/Schumann war der Anlass für das von Rebecca Grotjahn geleitete Symposium „Die Begleiterin - Clara Schumann, Lied und Liedinterpretation", das in enger Kooperation mit der Hochschule für Musik Detmold durchgeführt wurde. Das Hauptsymposium „Brückenschläge" wird in einem separaten Band publiziert (Bd. 3 der vorliegenden Reihe). Im Rahmen dieses Symposiums führte die von Stefanie Acquavella-Rauch geleitete Fachgruppe Digitale Musikwissenschaft eine Posterpräsentation durch, die von den Beiträger*innen erfreulicherweise zu kürzeren Texten umgearbeitet wurden, sodass sie hier ebenfalls, zusammen mit den Postern,

publiziert werden können. Hinzu kommen einige Beiträge, die bereits bei der Jahrestagung 2018 in Osnabrück präsentiert wurden. Auch das Hauptsymposium "Die Begleiterin" wird in einem eigenen Band (Bd. 2) publiziert. Die Beiträge zu den beiden anderen Hauptsymposien hingegen werden an anderen Orten veröffentlicht; in Band 1 („Freie Beiträge zur Jahrestagung der Gesellschaft für Musikforschung 2019") der vorliegenden Publikation finden sich jedoch Einführungen und Abstracts. Das Symposium „Komponieren für das Radio" unter Leitung von Antje Tumat und Camilla Bork (Katholieke Universiteit Leuven) behandelte Einflüsse des Mediums auf Kompositionsprozesse sowie durch radiophone Kompositionen bzw. radiophonen Klang ausgelöste Diskurse. Sarah Schauberger und Cornelia Bartsch (Universität Oldenburg) nahmen das 25-jährige Jubiläum der Fachgruppe Frauen- und Genderstudien zum Anlass für einen Generationenaustausch zum Thema "Musikwissenschaft - Feminismus - Kritik": Was wa- 
ren vor einem Vierteljahrhundert Inhalte und Aufgaben einer feministischen Musikwissenschaft und wie kann sich diese heute positionieren?

Bewusst haben wir im Tagungsbericht auf inhaltliche Eingriffe in die Beiträge verzichtet. ${ }^{1}$ Das gilt besonders für die Freien Referate: Es galt, den Charakter der Jahrestagung als Forum für ,freie', d. h. innovative und auch experimentelle Gedanken zu wahren. Einige Kolleg*innen, die die Tagung mit Vorträgen und Posterpräsentationen bereichert hatten, haben sich gegen eine Publikation im vorliegenden Band entschieden - sei es, weil sie eine Möglichkeit fanden, ihre Beiträge in einem inhaltlich passenderen Rahmen zu veröffentlichen, sei es, weil ihre Überlegungen in ihre entstehenden Qualifikationsschriften fließen sollen, oder sei es, weil sie von den Autor*innen in der vorgetragenen Form zunächst verworfen wurden. Auch damit erfüllt eine Freie-Referate-Sektion ihren Zweck: Die Diskussionen mit der versammelten Fach-Öffentlichkeit sollen dabei helfen, Gedanken weiterzuentwickeln und zu verändern. In diesem Sinne sei allen Beteiligten - den Autor*innen, den nichtpublizierenden Referent*innen und den Mit-Diskutant*innen - ganz herzlich gedankt für ihr Mitwirken bei der Tagung.

Unser herzlicher Dank gilt einer Reihe weiterer Personen, die zum Gelingen dieser drei Bände beigetragen haben. Hier ist besonders Jonas Spieker zu nennen, der uns tatkräftig bei der Redaktion geholfen hat. Andrea Hammes (SLUB Dresden) sei herzlich für die Aufnahme unseres Bandes auf musiconn.publish gedankt - wir freuen uns, damit unsererseits zur Etablierung dieser innovativen Publikationsplattform beizutragen.

Erneut möchten wir an dieser Stelle allen Menschen danken, die uns bei der Organisation, Ausrichtung und Finanzierung der Tagung selbst unterstützt haben: der Präsidentin der Universität Paderborn, Prof. Dr. Birgitt Riegraf, dem Rektor der Hochschule für Musik Detmold, Prof. Dr. Thomas Grosse, den Kolleginnen und Kollegen der beiden beteiligten Hochschulen, dem Vorstand der Gesellschaft für Musikforschung, der Universitätsgesellschaft Paderborn und allen Sponsoren. Besonders dankbar sind wir den Mitarbeiter*innen und den studentischen bzw. wissenschaftlichen Hilfskräften des Musikwissenschaftlichen Seminars, die bei der Vorbereitung und Ausrichtung der Tagung immensen Einsatz zeigten - stellvertretend sei an dieser Stelle Johanna Imm erwähnt, die zusammen mit Nina Jaeschke das Herz des Organisationsteams bildete.

Wir widmen diese Reihe Dr. Gabriele Buschmeier, dem langjährigen Vorstandsmitglied der Gesellschaft für Musikforschung, die kurz vor der Publikation dieses Bandes unerwartet verstarb.

Detmold, im September 2020

Rebecca Grotjahn und Nina Jaeschke

Zitation: Rebecca Grotjahn und Nina Jaeschke, „Vorwort”, in: Freie Beiträge zur Jahrestagung der Gesellschaft für Musikforschung 2019, hrsg. von Nina Jaeschke und Rebecca Grotjahn (= Musikwissenschaft: Aktuelle Perspektiven. Bericht über die Jahrestagung der Gesellschaft für Musikforschung 2019 in Paderborn und Detmold, Bd. 1), Detmold 2020, S. IX-X, DOI: 10.25366/2020.43.

1 Freigestellt war den Autor*innen auch, ob sie sich für eine gendersensible Sprache entscheiden bzw. welche Form des Genderns sie bevorzugen. 